\title{
Multiple bile-duct hamartomas (Von Meyenburg complexes)
}

\author{
M. C. Bailador Andrés, S. Vivas Alegre and R. Rueda Castañón ${ }^{1}$ \\ Services of Digesgestive Diseases and 'Radiodiagnosis. Hospital de León. Spain
}

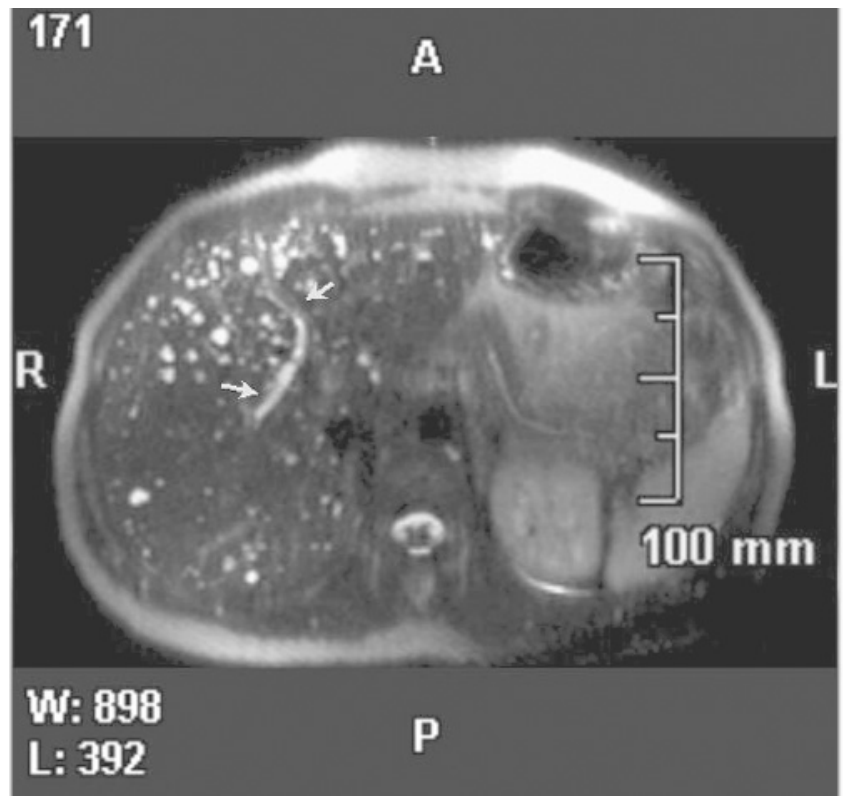

Fig. 1A.

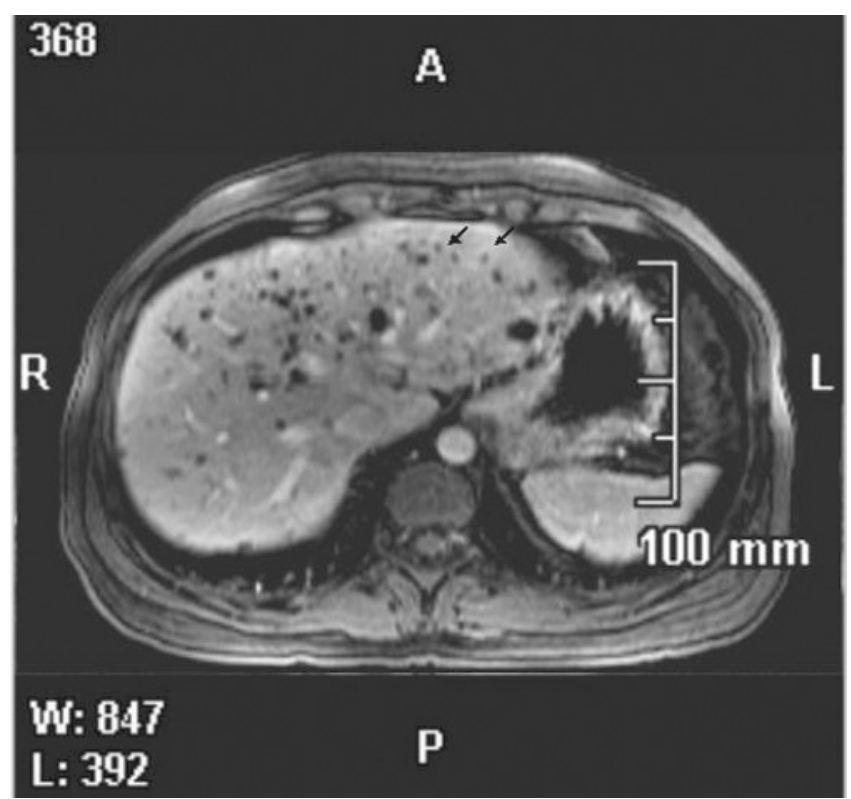

Fig. 1C.

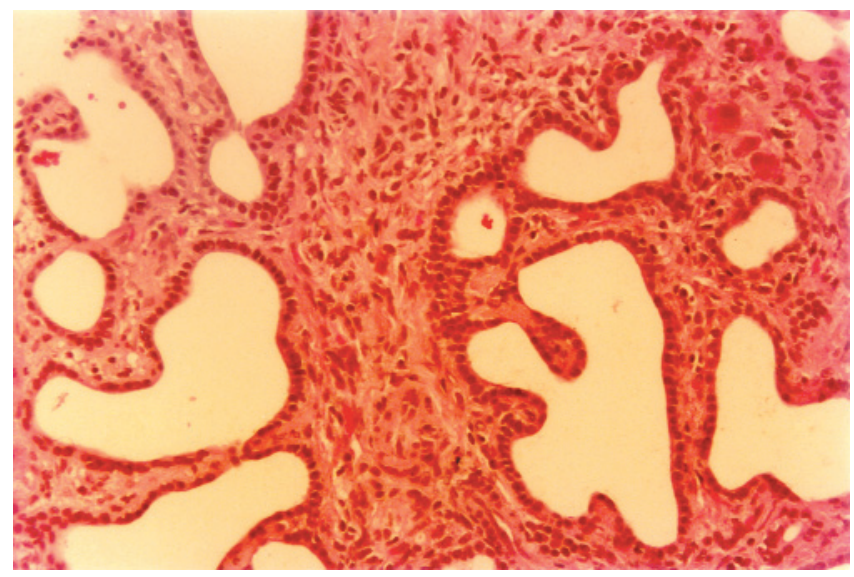

Fig. 1B.

An abdominal ultrasonography was performed for lumbar pain and showed multiple small hepatic nodules in a previously asymptomatic 37-year-old woman. Liver function tests and tumor markers were normal. Magnetic resonance imaging (MRI) demonstrated multiple highly intense, regular nodules (less than $15 \mathrm{~mm}$ ) with no communication to the bile tree (Fig. 1A). After intravenous gadolinium DTPA, some nodules showed peripheral enhancement from hepatic parenchymal compression around the nodules (Fig. 1B). These findings suggested hepatic bile-duct hamartomas, and the diagnosis was confirmed by liver biopsy (Fig. 1C).

Bile-duct hamartomas were described by Von Meyenburg in 1918. They are a ductal plate malformation at a late embryogenic stage, during which peripheral bile ducts grow, and may be included among liver fibropolycystic diseases (1). Hamartomas are characterized by dilated small bile ducts or cystically dilated bile ducts lined with epithelium and embedded in a fibrous stroma.

Ultrasonography usually shows multiple nodules with varying echogenicity scattered through both liver lobes. Computed tomography (CT) demonstrates multiple, irregular, small areas with scarce attenuation that usually show no enhancement after contrast injection. They can simulate 
abscesses, metastases, or Caroli's disease. MRI shows more pathognomonic findings versus CT, as reported in this case (2).

Microhamartomas are often asymptomatic, and usually represent casual findings. When multiple, serum gamma-glutamyltranspeptidase may rise, and very unusually a peripheral cholangiocarcinoma has been described in association with hamartomas (3), suggesting that malignant change may be possible.

\section{REFERENCES}

1. Desment VJ. Congenital diseases of intrahepatic bile ducts: variation on the theme "ductal plate malformation". Hepatology 1992; 16: 1069-83.

2. Cheung YC, Tan CF, Wan YL, Lui KW, Sai CC. MRI of multiple biliary hamartomas. Br J Radiol 1997; 70: 527-9.

3. Röcken C, Pross M, Brucks U, Ridwelski K, Roessmer A. Cholangiocarcinoma associated with multiple bile duct hamartomas. Arch Pathol Lab Med 2000; 124: 1704-6. 